\title{
Intravenous paracetamol versus ketorolac for pain attenuation in patients with renal colic; a randomized, single blind and controlled clinical trial
}

\author{
Arash Ardestani Zadeh ${ }^{1,2}$, Davood Arab $^{1,2^{*}}{ }^{\circledR}$, Mohammadreza Moonesan ${ }^{3}$, Majid Mirmohammadkhani $^{4}$, \\ Pouya Morid ${ }^{1}$
}

${ }^{1}$ Clinical Research Development Center, Kowsar Hospital, Semnan University of Medical Sciences, Semnan, Iran

${ }^{2}$ Department of Surgery, Kowsar Hospital, Semnan University of Medical Sciences, Semnan, Iran

${ }^{3}$ Department of Emergency Medicine, Kosar Hospital, Semnan University of Medical Sciences, Semnan, Iran

${ }^{4}$ Social Determinants of Health Research Center, Semnan University of Medical Sciences, Semnan, Iran

\section{A R T I C L E I N F O}

\section{Article Type:}

Original

\section{Article History:}

Received: 30 July 2019

Accepted: 24 December 2019

ePublished: 17 January 2020

\section{Keywords:}

Renal colic

Ketorolac

Paracetamol

Pain management

\begin{abstract}
A B S T R A C T
Introduction: Pain control is an essential care for patients with renal colic in emergency wards.

Objectives: This study aimed to compare the analgesic efficacy of intravenous (IV) paracetamol (PC) versus ketorolac (KET) for patients with renal colic.

Patients and Methods: In a randomized controlled clinical trial, 110 patients with renal colic referred to the emergency department of Kosar hospital, Semnan between October 2015 and June 2016 were selected. Eighty-eight patients were divided into two groups (44 patients in each group) of PC $(1 \mathrm{~g} / \mathrm{IV})$ and KET $(30 \mathrm{mg} / \mathrm{IV})$. One patient in each group was excluded during the study. Vital signs and pain severity (measured by visual analogue scale [VAS]) of all patients were recorded at admission time 0, 20, 40 and 60 minutes after treatment. Then, the results were compared in two groups.

Results: The results showed that at the time of $0,20,40$ and 60 minutes after the administration of the PC and KET drugs, no significant difference was seen in severity of pain based on VAS score between the two groups $(P<0.05)$. Moreover, there were no significant differences in the vital signs of two groups $(P<0.05)$. No adverse effects were reported in each group.

Conclusion: In conclusion, the use of IV-PC and KET in patients with renal colic had similar pain relieving effects without any adverse effects.

Trial Registration: The study was registered at Iranian Registry of Clinical Trials (identifier: IRCT2015090223855NI; http://irct.ir/trial/20282).
\end{abstract}

Implication for health policy/practice/research/medical education:

In a randomized controlled clinical trial on 110 patients with renal colic, we found the similar efficacy in pain relieving for paracetamol versus ketorolac.

Please cite this paper as: Ardestani Zadeh A, Arab D, Moonesan M, Mirmohammadkhani M, Morid P. Intravenous paracetamol versus ketorolac for pain attenuation in patients with renal colic; a randomized, single blind and controlled clinical trial. J Renal Inj Prev. 2020; 9(2): e10. doi: 10.34172/jrip.2020.10.

\section{Introduction}

Renal colic usually presents as an intermittent and severe flank pain (1). The prevalence of renal colic is thought to be between $2 \%$ to $3 \%$. In recent years, the incidence of renal colic has been increasing due to alterations in individuals' diet and lifestyle (2). As a most painful conditions, renal colic require urgent pain relief treatment (3). Renal colic pain is characterized by a sudden intense, sharp and bothering pain located in the flank or abdominal area (4).
This commonly happens when a urinary stone obstructs the ureter. The main goal of managements in emergency department is prompt pain relief (5).

Different types of pain killers are used to ease the discomfort. Non-steroidal anti-inflammatory drugs (NSAIDs) and opioids are commonly used as pain relievers in emergency department. However, both of these classes of drugs have many side effects (6). In a meta-analysis, NSAIDs were found to be as effective as different types 
of analgesic agents such as narcotics in treatment of acute renal colic (7). Ketorolac (KET), a NSAID, is suggested for pain management, inflammation and fever. However, reduced narcotic usage and side effects were reported for this agent (8). KET has been demonstrated to induce platelet dysfunction and increased incidence of hematoma after surgery $(9,10)$.

Recently, the Food and Drug Administration (FDA) approved that intravenous (IV) paracetamol (PC) or acetaminophen, a non-NSAID analgesic and anti-pyretic agent obtains more effective onset and faster outcome compared to rectally or orally administer PC (11). Studies investigating the impacts of PC in pain relief of patients with renal colic, showed that PC might be an effective agent as compared to opioids $(3,12)$. It was shown that the analgesic effect of IV-PC is induced by cyclooxygenase and serotonin system inhibition and this makes it a safe analgesic drug. Also, this agent passes through the blood brain barrier and reaches a high concentration in the central nervous system (13).

\section{Objectives}

This study aimed to compare the effectiveness of intravenous PC versus KET in pain relief of patients with renal colic pain.

\section{Patients and Methods}

\section{Study design}

In a randomized, single blind and controlled clinical trial, 110 patients with renal colic (18 to 55 years) referred to emergency ward of Kosar hospital, affiliated to Semnan University of Medical Sciences, Semnan, Iran, were enrolled into the study and 86/110 cases were investigated between October 2015 and June 2016.

\section{Participants}

This study was conducted on 110 patients who were presented to emergency ward with chief complaint of flank pain and highly suspected renal colic. The inclusion criteria included all patients with acute severe flank pain that radiated to abdomen or ipsilateral groin. Visual analogue scale (VAS) is a measurement instrument scored 0 (no pain) to 10 (the worst possible pain). Urinary stone was proven by clinical investigation and/ or ultrasonography, CT scan or intravenous pyelography. The pregnant women and patients with history of allergy to NSAIDs, asthma, renal failure, fever, gastrointestinal bleeding and recent use of analgesics were excluded. All evaluations were performed by an emergency medicine specialist. Twenty-two cases were not enrolled into the study due to the exclusion criteria.

\section{Intervention}

In this study 88 cases selected using a convenience sampling and divided into two groups (44 in each group) of study using permuted balanced block randomization (Figure 1). In the present study, permuted block randomization was used to allocate interventions in a completely random manner to the two treatment groups. Six blocks of four were defined. Structure of each block was four-way

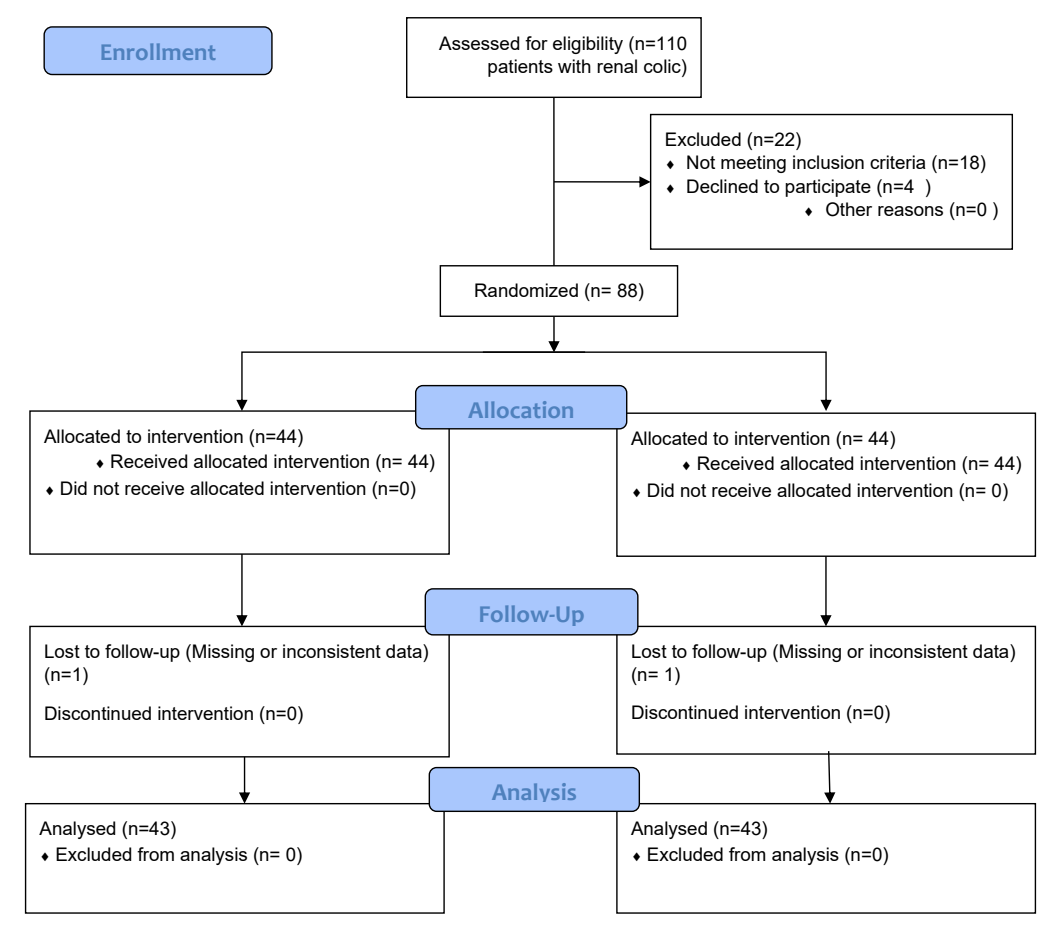

Figure 1. CONSORT flow diagram. 
combination of two methods of intervention in a perfectly balanced way. Random digits table was used for random assignment of blocks to each group. Accordingly, a list was prepared and eligible patients were enrolled in the study according to the list, respectively. Additional matching did not take place and one of the authors planned for allocation before starting the investigation. Patients and data analysts did not know about the treatment allocation. The groups of study were defined as follows:

- $\quad$ PC group ( $n=44)$ : Patients received intravenous (IV) 1 gr PC solution (Apotel, $10 \mathrm{mg} / \mathrm{mL}$ Sol. IV. Inf., Uni-Pharma) dissolved in $100 \mathrm{~mL}$ normal saline and infused in 15 minutes.

- KET group ( $n=44)$ : Patients received IV $30 \mathrm{mg}$ ketorolac tromethamine infused in 15 seconds (Alborz-Darou Co. Qazvin).

The pain severity was examined using VAS measurement instrument in different time points of admission time (0), 20, 40 and 60 minutes after drugs administration. In addition to pain other factors including axillary temperature, systolic and diastolic pressure, heart rate, respiratory rate of patients and the side effects of drugs were investigated in these time points. In this way, a checklist was designed to collect the data required to make the assessments. The main objective was to compare the VAS scores at different times of 20, 40, 60 minutes after treatment. Data of 43 patients in each group were analyzed due to missing or inconsistent data $(n=1$ in PC group and $n=1$ in KET group). As well, if the pain severity did not reduce by $50 \%$, opioid was used to relieve the pain (Figure 2).

\section{Ethical issues}

The research followed the tenets of the Declaration of Helsinki. All patients signed a written informed consent form to participate in the study. The Ethics Committee of Semnan University of Medical Sciences approved the study in August 2015 (registration code\#IR.SEMUMS.REC.1394.72). The study was also registered at Iranian Registry of Clinical Trials (identifier: IRCT2015090223855NI; http://irct.ir/trial/20282). This study was extracted from M.D, thesis of Pouya Morid (Thesis\# 686; proposal \# A-10-140-4) at this university.

\section{Statistical analysis}

Data were analyzed using SPSS 22. The differences in the quantitative variables were determined using $t$ test or Mann Whitney U test, and qualitative and differences in the numerical variables were evaluated by chi-square and Fisher's exact tests. $P<0.05$ was considered significant.

\section{Results}

In this study, 88 patients in two treatment groups of KET and PC were investigated. Two cases were excluded due to missing or inconsistent data $(\mathrm{n}=1$ in the PC group and $\mathrm{n}=1$ in the KET group) during the study and data of 86
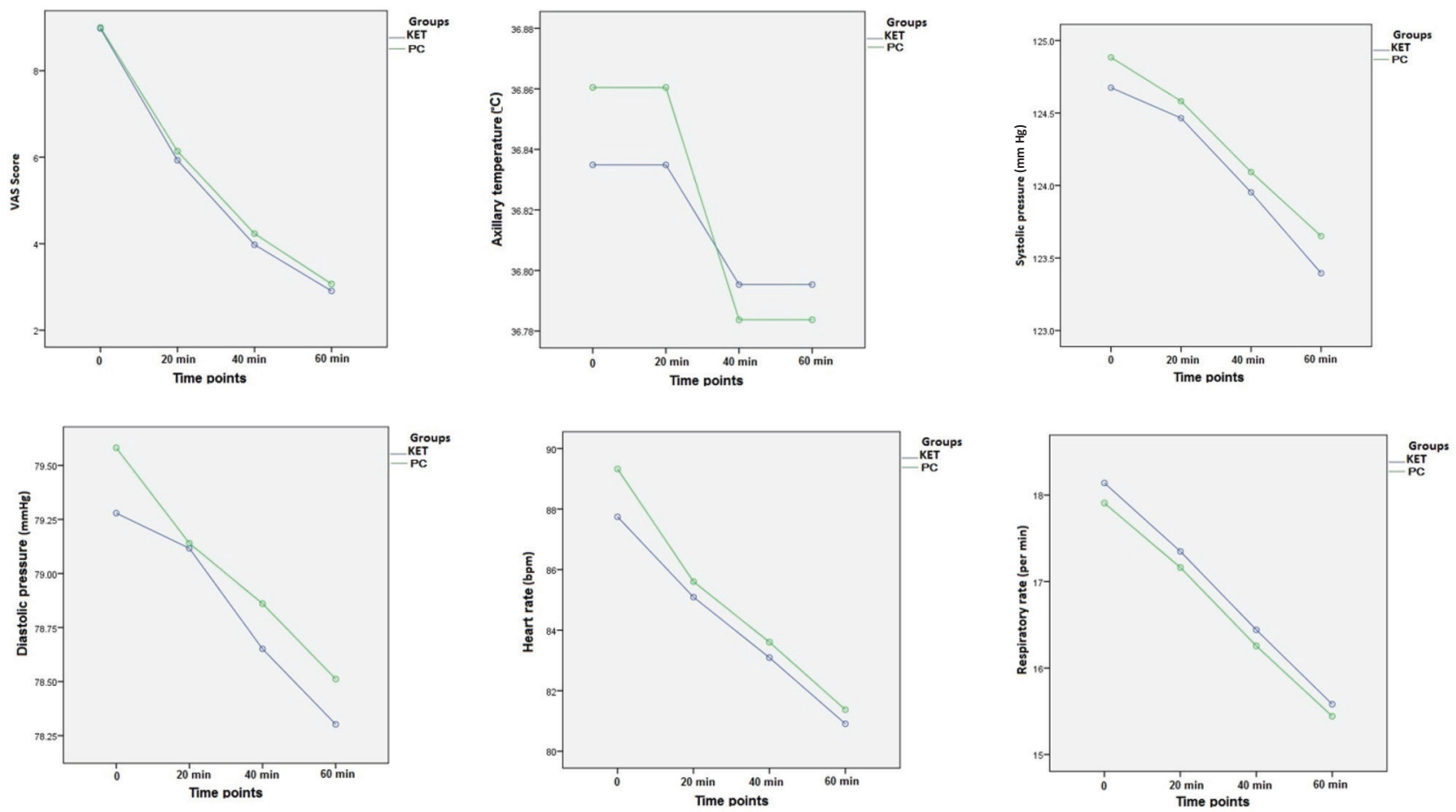

Figure 2. Comparing the vital signs and pain severity between two groups based on different time points including admission time $0,20,40$ and 60 minutes after drugs administration. 
Table 1. Comparing the different characteristics of patients with renal colic based on study groups

\begin{tabular}{|c|c|c|c|}
\hline & \multicolumn{3}{|c|}{ Groups } \\
\hline & \multirow{2}{*}{$\begin{array}{c}\text { KET } \\
\text { No. }(\%) \text { or Mean } \pm \text { SD* (range) }\end{array}$} & \multirow{2}{*}{$\begin{array}{c}\text { PC } \\
\text { No. }(\%) \text { or Mean } \pm \text { SD (range) }\end{array}$} & \multirow{2}{*}{$P$ value } \\
\hline & & & \\
\hline Age (y) & $38.07 \pm 7.97$ (20 to 54$)$ & $36.58 \pm 5.62(27-47)$ & 0.181 \\
\hline Gender (male) & $33(76.7 \%)$ & $32(74.4 \%)$ & 0.802 \\
\hline Diabetes & $1(2.3 \%)$ & $2(4.7 \%)$ & 0.5 \\
\hline Hematuria & $15(34.9 \%)$ & $15(34.9 \%)$ & 1 \\
\hline Pyuria & $3(7 \%)$ & $2(4.7 \%)$ & 0.5 \\
\hline Diameter of stones (mm) & $7.05 \pm 1.99(3-12)$ & $6.14 \pm 1.42(5-10)$ & 0.273 \\
\hline \multicolumn{4}{|l|}{ Areas } \\
\hline Left pelvis and UPJ & $7(46.7 \%)$ & $8(53.3 \%)$ & 0.771 \\
\hline Right pelvis and UPJ & $8(53.3 \%)$ & $7(46.7 \%)$ & 0.776 \\
\hline Right upper ureter & $8(42.1 \%)$ & $11(57.9 \%)$ & 0.432 \\
\hline Right lower ureter & $6(75 \%)$ & $2(25 \%)$ & 0.138 \\
\hline Left upper ureter & $8(50 \%)$ & $8(50 \%)$ & 1 \\
\hline Left lower ureter & $6(46.2 \%)$ & $7(53.8 \%)$ & 0.763 \\
\hline History of renal colic & $22(56.4 \%)$ & $17(43.6 \%)$ & 0.279 \\
\hline History of urinary stones & $31(57.4 \%)$ & $23(42.6 \%)$ & 0.074 \\
\hline Opioids for pain relief & $11(25.6 \%)$ & 9 (20.9\%) & 0.425 \\
\hline
\end{tabular}

SD, Standard deviation; UPJ, ureteropelvic junction.

patients were analyzed. The demographic characteristics of patients in the two groups were compared in Table 1. Mean age of KET group was $38.07 \pm 7.97$ years (range of 20 to 54 years) and the PC group was $36.58 \pm 5.62$ years (range of 27 to 47 years). No significant differences were observed in the age of two groups (Table $1, P=0.181$ ). Thirty-three patients $(76.7 \%)$ in the KET group and $32(74.4 \%)$ in the PC group were male. In addition, the distribution of cases with diabetes, hematuria and pyuria were investigated based on two groups of study. There were no significant differences in the distribution of the gender (Table 1, $P=0.802$ ), diabetes (Table $1, P=0.5$ ), hematuria (Table 1, $P=1$ ) and pyuria (Table $1, P=0.5$ ) of cases in two groups of study. There were no significant differences in the mean diameter of urinary stone in two groups (Table 1, $P=0.273$ ). Moreover, no significant difference of distribution of urinary stones between the two groups was observed (Table 1). Furthermore, the history of renal colic and urinary stones and the need to opioids for pain relief were compared between the two groups (Table 1).

According to Table 2 and Figure 2, the mean VAS score was evaluated in the two groups. No significant differences were observed in VAS score of the two groups based on different time points of $0(P=0.927), 20$ $(P=0.625), 40(P=0.623)$ and $60(P=0.804)$ minutes after treatment. Additionally, no significant differences in the mean axillary temperature $(P=0.328, P=0.328, P=0.647$ and $P=0.512$, respectively), systolic pressure $(P=0.929$, $P=0.96, P=0.951$ and $P=0.907$, respectively), diastolic pressure $(P=0.848, P=0.988, P=0.889$ and $P=0.888$, respectively), heart rate $(P=0.3, \mathrm{P}=0.309, P=0.889$ and $P=0.556$, respectively) and respiratory rate $(P=0.274$,
$P=0.309, P=0.251$ and $P=0.443$, respectively) of patients in two groups in different time points of $0,20,40$ and 60 minutes after treatment were reported (Table 1 and Figure 2).

\section{Discussion}

The present study compared the impacts of IV-PC and IV KET on renal colic pain. The results of data analysis showed that both groups were similar in the different characteristics such as age, gender, diabetes, hematuria, pyuria, stone diameter and location, history of renal colic, and urinary stones. Pain management is one of the critical cares in patients who present with renal colic in the emergency ward (14). NSAIDs and opioids have been reported to be the main classes of drugs for pain relief in patients with renal colic. However, different side effects have been reported for these drugs (15). Moreover, evidence suggested that frequently use of KET is related to enhanced risk of gastrointestinal bleeding (8).

IV-PC is a non-NSAID analgesic drug, which is widely used for pain relief in a emergency department $(16,17)$. Based on the findings of present study, PC and KET had equal effectiveness regarding renal colic pain management with no reported side effects. According to the literature, the PC efficiency was compared to other drugs when they were administered for pain relief in patients with renal colic.

The findings of this study are in accordance with previous studies. In similar research, Morgan et al, the effectiveness, mechanism of action and pharmacokinetics of IV-PC on renal colic was evaluated and suggested that all prescribers can use this drug to quickly relieve the pain in these 
Table 2. Comparing the vital signs and pain severity between two groups based on different time points including admission time (0), 20 min, 40 min and 60 min after drugs administration

\begin{tabular}{|c|c|c|c|c|}
\hline & & \multicolumn{3}{|c|}{ Groups } \\
\hline & & KET & PC & P value \\
\hline & & Mean $\pm S D$ & Mean $\pm S D$ & $P$ value \\
\hline \multirow{4}{*}{ VAS score } & 0 & $8.98 \pm 1.3$ & $9 \pm 1.05$ & 0.927 \\
\hline & 20 min after treatment & $5.93 \pm 2.16$ & $6.14 \pm 1.77$ & 0.625 \\
\hline & 40 min after treatment & $3.98 \pm 2.6$ & $4.23 \pm 2.19$ & 0.623 \\
\hline & 60 min after treatment & $2.91 \pm 3.05$ & $3.07 \pm 3$ & 0.804 \\
\hline \multirow{4}{*}{ Axillary temperature $\left({ }^{\circ} \mathrm{C}\right)$} & 0 & $36.83 \pm 0.12$ & $36.86 \pm 0.12$ & 0.328 \\
\hline & 20 min after treatment & $36.84 \pm 0.12$ & $36.86 \pm 0.12$ & 0.328 \\
\hline & 40 min after treatment & $36.79 \pm 0.32$ & $36.78 \pm 0.13$ & 0.647 \\
\hline & 60 min after treatment & $36.79 \pm 0.15$ & $36.77 \pm 0.09$ & 0.512 \\
\hline \multirow{4}{*}{ Systolic pressure (mm Hg) } & 0 & $124.67 \pm 11.12$ & $124.88 \pm 10.73$ & 0.929 \\
\hline & 20 min after treatment & $124.47 \pm 10.88$ & $124.58 \pm 10.56$ & 0.960 \\
\hline & 40 min after treatment & $123.95 \pm 10.69$ & $124.09 \pm 10.27$ & 0.951 \\
\hline & 60 min after treatment & $123.4 \pm 10.3$ & $123.65 \pm 9.9$ & 0.907 \\
\hline \multirow{4}{*}{$\begin{array}{l}\text { Diastolic pressure } \\
(\mathrm{mm} \mathrm{Hg})\end{array}$} & 0 & $79.28 \pm 7.11$ & $79.58 \pm 7.45$ & 0.848 \\
\hline & 20 min after treatment & $77.12 \pm 7.03$ & $77.14 \pm 7.2$ & 0.988 \\
\hline & 40 min after treatment & $78.65 \pm 6.68$ & $78.86 \pm 7.17$ & 0.889 \\
\hline & 60 min after treatment & $78.3 \pm 6.7$ & $78.51 \pm 7.08$ & 0.888 \\
\hline \multirow{4}{*}{ Heart rate (bpm) } & 0 & $87.74 \pm 1.99$ & $89.33 \pm 2.16$ & 0.3 \\
\hline & 20 min after treatment & $85.09 \pm 2.57$ & $85.6 \pm 3.79$ & 0.309 \\
\hline & 40 min after treatment & $83.09 \pm 2.55$ & $83.6 \pm 3.8$ & 0.889 \\
\hline & 60 min after treatment & $80.91 \pm 3.08$ & $81.37 \pm 4.13$ & 0.556 \\
\hline \multirow{4}{*}{ Respiratory rate (per minute) } & 0 & $18.14 \pm 0.86$ & $17.91 \pm 1.09$ & 0.274 \\
\hline & 20 min after treatment & $17.35 \pm 0.81$ & $17.35 \pm 0.87$ & 0.309 \\
\hline & 40 min after treatment & $16.44 \pm 0.7$ & $16.26 \pm 0.79$ & 0.251 \\
\hline & 60 min after treatment & $15.58 \pm 0.82$ & $15.44 \pm 0.85$ & 0.443 \\
\hline
\end{tabular}

SD, Standard deviation.

patients (18). To compare the PC with NSAID drugs, Grissa et al estimated the effectiveness of IV-PC versus IM (intra-muscular) piroxicam and showed that the pain relieved in $80 \%$ of patients in PC group and only $48 \%$ in piroxicam group after 90 minutes (19).

To compare the IV-PC with morphine for the management of acute renal colic pain, Bektas et al conducted a doubleblind, placebo-controlled clinical trial and showed that the efficacy of both IV-PC and morphine was similar (12). In a systematic review and meta-analysis by Sin et al, the safety, efficacy, cost-benefits and opioid-sparing effects of IV-PC in patients with renal colic were determined and demonstrated that IV-PC was weaker in the pain reduction than opioids or nonsteroidal anti-inflammatory drugs (20).

Additionally, different studies evaluated the side effects of PC versus KET following different procedures. Rusy et al investigated the efficacy and side effects (such as bleeding) of KET versus high-dose rectal PC in the patient undergoing tonsillectomy in a double-blind study and proved that KET was not more effective than high-dose rectal PC for analgesia in these patients. On the other hand, hemostasis during tonsillectomy was difficultly achieved in patients received KET (21). Additionally, KET-induced acute renal failure has been shown in a healthy adolescent (22).

\section{Conclusion}

Our findings indicated the similar efficacy in pain relieving for both PC and KET. Due to the availability of PC in emergency wards, the results of present study suggest that PC can be used instead of KET for renal colic pain management.

\section{Limitations of the study}

The limitation of this study was the possibility of examining other factors related to changes in pain severity.

\section{Authors' contribution}

DA and AA conducted the research. MM, MM and PM helped to prepare the manuscript. DA and AA prepared the final manuscript. All authors read and signed the final paper.

\section{Conflicts of interest}

The authors declared no competing interests. 
Ethical considerations

Ethical issues (including plagiarism, data fabrication, double publication) have been completely observed by the authors.

\section{Funding/Support}

This study was supported by Semnan University of Medical Sciences. This study was extracted from M.D, Thesis of Pouya Morid (Thesis\# 686 and proposal \# A-10140-4).

\section{References}

1. Holdgate A, Pollock T. Systematic review of the relative efficacy of non-steroidal anti-inflammatory drugs and opioids in the treatment of acute renal colic. BMJ. 2004;328:1401. doi: 10.1136/bmj.38119.581991.55.

2. Romero V, Akpinar H, Assimos DG. Kidney stones: a global picture of prevalence, incidence, and associated risk factors. Rev Urol. 2010;12:e86.

3. Serinken M, Eken C, Turkcuer I, Elicabuk H, Uyanik E, Schultz CH. Intravenous paracetamol versus morphine for renal colic in the emergency department: a randomised double-blind controlled trial. Emerg Med J. 2012;29:902-5. doi: 10.1136/emermed-2011-200165.

4. Cox C, MacDonald S, Henneberry R, Atkinson PR. My patient has abdominal and flank pain: identifying renal causes. Ultrasound. 2015;23:242-50. doi: $10.1177 / 1742271 \mathrm{X} 15601617$.

5. Osorio L, Lima E, Autorino R, Marcelo F. Emergency management of ureteral stones: Recent advances. Indian J Urol. 2008;24:461-6. doi: 10.4103/0970-1591.44248.

6. Pathan SA, Mitra B, Romero L, Cameron PA. What is the best analgesic option for patients presenting with renal colic to the emergency department? Protocol for a systematic review and meta-analysis. BMJ Open. 2017;7:e015002-e. doi: 10.1136/bmjopen-2016-015002.

7. Labrecque M, Dostaler L-P, Rousselle R, Nguyen T, Poirier S. Efficacy of nonsteroidal anti-inflammatory drugs in the treatment of acute renal colic: a meta-analysis. Arch Intern Med. 1994;154:1381-7.

8. Gillis JC, Brogden RN. Ketorolac. A reappraisal of its pharmacodynamic and pharmacokinetic properties and therapeutic use in pain management. Drugs. 1997;53:13988 .

9. Chin CJ, Franklin JH, Turner B, Sowerby L, Fung K, Yoo $\mathrm{JH}$. Ketorolac in thyroid surgery: quantifying the risk of hematoma. J Otolaryngol Head Neck Surg. 2011;40:196-9.

10. Niemi TT, Backman JT, Syrjala MT, Viinikka LU, Rosenberg PH. Platelet dysfunction after intravenous ketorolac or propacetamol. Acta Anaesthesiol Scand. 2000;44:69-74. doi: 10.1034/j.1399-6576.2000.440113.x.

11. Brett CN, Barnett SG, Pearson J. Postoperative plasma paracetamol levels following oral or intravenous paracetamol administration: a double-blind randomised controlled trial. Anaesth Intensive Care. 2012;40:166-71. doi: $\quad 10.1177 / 0310057 X 1204000121$.

12. Bektas F, Eken C, Karadenız O, Goksu E, Cubuk M, Cete Y. Intravenous paracetamol or morphine for the treatment of renal colic: a randomized, placebo-controlled trial. Ann Emerg Med. 2009;54:568-74. doi: 10.1016/j. annemergmed.2009.06.501.

13. Remy C, Marret E, Bonnet F. State of the art of paracetamol in acute pain therapy. Curr Opin Anaesthesiol. 2006;19:5625. doi: 10.1097/01.aco.0000245285.30282.70.

14. Turkcuer I, Serinken M, Karcioglu O, Zencir M, Keysan MK. Hospital cost analysis of management of patients with renal colic in the emergency department. Urol Res. 2010;38:29-33. doi: 10.1007/s00240-009-0251-z.

15. Pathan SA, Mitra B, Romero L, Cameron PA. What is the best analgesic option for patients presenting with renal colic to the emergency department? Protocol for a systematic review and meta-analysis. BMJ Open. 2017;7:e015002. doi: 10.1136/bmjopen-2016-015002. doi: 10.1136/ bmjopen-2016-015002.

16. Furyk J, Levas D, Close B, Laspina K, Fitzpatrick M, Robinson $\mathrm{K}$, et al. Intravenous versus oral paracetamol for acute pain in adults in the emergency department setting: a prospective, double-blind, double-dummy, randomised controlled trial. Emerg Med J. 2018;35:179-84. doi: 10.1136/ emermed-2017-206787.

17. Barnaby DP, Chertoff AE, Restivo AJ, Campbell CM, Pearlman S, White D, et al. Randomized Controlled Trial of Intravenous Acetaminophen Versus Intravenous Hydromorphone for the Treatment of Acute Pain in the Emergency Department. Ann Emerg Med. 2019;73:13340. doi: 10.1016/j.annemergmed.2018.06.019.

18. Morgan S. Intravenous paracetamol in patients with renal colic. Emerg Nurse. 2011;18:22-5. doi: 10.7748/ en2011.02.18.9.22.c8337.

19. Grissa MH, Claessens YE, Bouida W, Boubaker H, Boudhib L, Kerkeni W, et al. Paracetamol vs piroxicam to relieve pain in renal colic. Results of a randomized controlled trial. Am J Emerg Med. 2011;29:203-6. doi: 10.1016/j. ajem.2009.09.019.

20. Sin B, Koop K, Liu M, Yeh JY, Thandi P. Intravenous Acetaminophen for Renal Colic in the Emergency Department: Where Do We Stand? Am J Ther. 2017;24:e12e19. doi: 10.1097/MJT.0000000000000526.

21. Rusy LM, Houck CS, Sullivan LJ, Jones DT, McGill TJ, Berde CB. A double-blind evaluation of ketorolac tromethamine versus acetaminophen in pediatric tonsillectomy: analgesia and bleeding. Anesth Analg. 1995;80:226-9.

22. Buck ML, Norwood VF. Ketorolac-induced acute renal failure in a previously healthy adolescent. Pediatrics. 1996;98:294-6.

Copyright (C) 2020 The Author(s); Published by Nickan Research Institute. This is an open-access article distributed under the terms of the Creative Commons Attribution License (http://creativecommons.org/licenses/by/4.0), which permits unrestricted use, distribution, and reproduction in any medium, provided the original work is properly cited. 